\title{
STATIC AND DYNAMIC ANALYSES OF COMPOSITE BEAMS WITH INTERLAYER SLIP
}

\author{
Ákos JózSEF LENGYel ANd István ECSEDI \\ Institute of Applied Mechanics, University of Miskolc \\ H-3515 Miskolc, Miskolc-Egyetemváros, Hungary \\ mechlen@uni-miskolc.hu, mechecs@uni-miskolc.hu
}

[Received: November 17, 2014, Accepted: January 16, 2015]

\begin{abstract}
The present paper gives analytical solutions for shear deformable two-layer beams with weak shear connections. Timoshenko's kinematic assumptions are applied to both layers with different cross-sectional rotations. A linear constitutive equation is used between the horizontal shear force and the interlayer slip. The applied loads act in the plane of symmetry of a two-layered beam and the material-geometrical properties do not depend on the axial coordinate. For simply supported beam closed form solutions are derived for the deflection, slip and cross-sectional rotations. The eigenfrequencies of a simply supported beam are determined and compared with the solutions obtained by the applications of Euler-Bernoulli and Euler-Bernoulli-Rayleigh beam models.
\end{abstract}

Mathematical Subject Classification: 74G05, 34B05

Keywords: interlayer slip, shear connection, composite beam, free vibration

\section{INTRODUCTION}

Layered beams made of different elastic materials are frequently used in construction and they have created a growing interest in the different engineering sectors where both high strength-to-weight and stiffness-to-weight ratio are desired. There are many ways to form a connection between layers made of different materials. In some cases it occurs that the connection is weak in shear permitting only a relative slip, but preserving the contact in normal direction. The problem of layered beams with deformable shear connections has been studied for a long time. The first theories for these composite beams were developed by Granholm [1], Pleskov 2, Stüssi [3] and Newmark et al. [4]. The static analysis done by Newmark et al. [4] is based on the Euler-Bernoulli beam theory and has become a basis for investigating layered beam systems with interlayer slip $5-22$. Today the analytical and numerical FEM solution are refined 5 26]. Several studies [5 8, 13 present FEM solutions for multilayer beams with weak shear connections using the Euler-Bernoulli beam theory. Exact first and second order static analyses for composite beam-columns with partial shear interaction subjected to transverse and axial loads are given by Girhammar and Gopu 9 . By using variational methods Girhammar and Pan 10 derive ordinary differential 
equations for the deflections and set up the corresponding boundary conditions for partially composite Euler-Bernoulli beams and beam-columns. A simplified analysis and design method for composite members with partial shear interaction that predicts the deflections and stresses has been proposed by Girhammar 11. In 16, 17,20 researchers developed FEM formulations for composite beams with deformable shear connection. The derived stiffness matrix takes the effects of interface slip and shear deformations into account. In [20] it is assumed that the cross-sectional rotations are not the same for the different beam components and the effect of shear connectors on a composite beam element is described by two springs which are separately placed at the two ends of the considered element.

Dynamic analysis of a composite beam with deformable shear connections based on the Euler-Bernoulli beam theory is presented by Girhammar and Gopu 23]. They consider free and forced vibrations. The governing differential equations and corresponding boundary conditions are derived for partial-interaction composite members and exact analytical solution for simply supported boundary conditions is presented in 25]. In paper 24 an analytical solution for free vibrations of shear-deformable two-layer beams with interlayer slip and axial load is developed. The effect of transverse shear flexibility of two layers is taken into account in a general way. Each layer behaves as a Timoshenko beam.

The present paper deals with two-layer beams with interlayer slip and gives analytical solutions for deflection, slip and cross-sectional rotations in the case of a static equilibrium problem. By introducing the inertia forces into the equilibrium equations we derive the equations of free vibrations as well. Applications of the equations we have established are illustrated via numerical examples.

\section{Governing EQUATions}

In the reference configuration a composite beam with two components occupies the cylindrical region $B=A \times[0, L]$ generated by translating its cross-section $A$ with a regular boundary $\partial A$ along a rectilinear axis, normal to the cross-section. The cross-section $A$ is divided into two parts $A_{1}$ and $A_{2}$ by the curve $\partial A_{12}$ describing the positions of continuous connection such that (see Figure 1)

$$
\begin{gathered}
B_{i}=A_{i} \times(0, L), \quad(i=1,2), \quad A=A_{1} \cup A_{2}, \quad B=B_{1} \cup B_{2}, \\
\partial A_{i}=\partial A_{i 0} \cup \partial A_{12}, \quad(i=1,2), \quad \partial A=\partial A_{10} \cup \partial A_{12} .
\end{gathered}
$$

Here $L$ is the length of the beam. A point $P$ in $\bar{B}=B \cup \partial B(\partial B$ is the boundary surface of $B$ ) is indicated by the position vector $\mathbf{r}=x \mathbf{e}_{x}+y \mathbf{e}_{y}+z \mathbf{e}_{z}$, where $x, y, z$ and $\mathbf{e}_{m}\left(\left|\mathbf{e}_{m}\right|=1, m=x, y, z\right)$ refer to a rectangular coordinate system Oxyz. The equation of the common boundary surface of the beam components $B_{1}$ and $B_{2}$ is $y=0,0 \leq z \leq L$ (see Figure 1). The center of $A_{i}$ is denoted by $C_{i}(i=1,2)$. The plane $y z$ is the plane of symmetry for the geometrical and material properties and loading conditions. According to Timoshenko's beam theory, which is valid for each homogeneous layer, the deformation of the beam is described by the following displacement field (see Figure 2) 

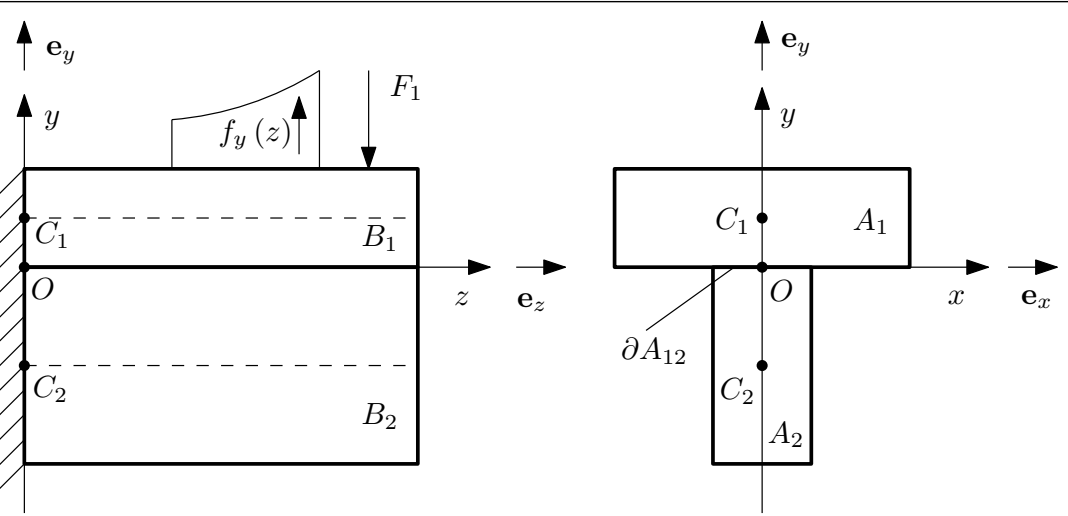

Figure 1. A composite beam with weak shear connection

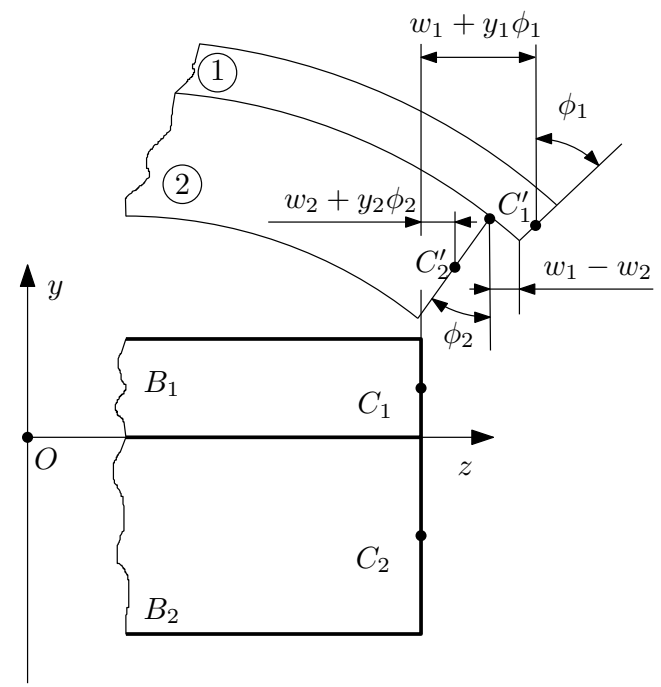

Figure 2. Kinematical model for a composite beam with interlayer slip

$$
\mathbf{u}=u(x, y, z) \mathbf{e}_{x}+v(x, y, z) \mathbf{e}_{y}+w(x, y, z) \mathbf{e}_{z},
$$

where

$$
u=0, \quad v=v(z), \quad w(y, z)=w_{i}(z)+y \phi_{i}(z), \quad(x, y, z) \in B_{i}, \quad(i=1,2) .
$$

In Figure $2 C_{i}^{\prime}(i=1,2)$ denotes the center of $A_{i}$ in the deformed configuration of the considered cross-section. On the common boundary of the beam component the axial displacement $w$ has a jump which is called an interlayer slip. From equation (2.4) and the definition of interlayer slip $s$ it follows that

$$
s(x, y, z)=w_{1}(z)-w_{2}(z), \quad y=0,0 \leq z \leq L .
$$


Application of the strain displacement relationships of the linearized theory of elasticity yields 27,28

$$
\begin{gathered}
\varepsilon_{x}=\varepsilon_{y}=\gamma_{x y}=\gamma_{x z}=0 \\
\varepsilon_{z}=\frac{\mathrm{d} w_{i}}{\mathrm{~d} z}+y \frac{\mathrm{d} \phi_{i}}{\mathrm{~d} z}, \quad(x, y, z) \in B_{i}, \quad(i=1,2), \\
\gamma_{y z}=\frac{\mathrm{d} v}{\mathrm{~d} z}+\phi_{i}, \quad(x, y, z) \in B_{i}, \quad(i=1,2),
\end{gathered}
$$

where $\varepsilon_{x}, \varepsilon_{y}, \varepsilon_{z}$ and $\gamma_{x y}, \gamma_{x z}, \gamma_{y z}$ are the axial and shearing strains. From Hooke's law we get the normal stress $\sigma_{z}$ and the shearing stress $\tau_{y z}$ in terms of strains as

$$
\begin{gathered}
\sigma_{z}=E_{i}\left(\frac{\mathrm{d} w_{i}}{\mathrm{~d} z}+y \frac{\mathrm{d} \phi_{i}}{\mathrm{~d} z}\right), \quad(x, y, z) \in B_{i}, \quad(i=1,2) \\
\tau_{y z}=G_{i}\left(\frac{\mathrm{d} v}{\mathrm{~d} z}+\phi_{i}\right), \quad(x, y, z) \in B_{i}, \quad(i=1,2) .
\end{gathered}
$$

In equations 2.9), 2.10 $E_{i}$ is the Young modulus and $G_{i}$ is the shear modulus. We introduce the following section forces and section moment

$$
\begin{gathered}
N_{i}=\int_{A_{i}} \sigma_{z} \mathrm{~d} A=A_{i} E_{i}\left(\frac{\mathrm{d} w_{i}}{\mathrm{~d} z}+y_{i} \frac{\mathrm{d} \phi_{i}}{\mathrm{~d} z}\right), \quad y_{i}=\frac{1}{A_{i}} \int_{A_{i}} y \mathrm{~d} A, \quad(i=1,2), \\
M_{i}=\int_{A_{i}} y \sigma_{z} \mathrm{~d} A=A_{i} E_{i}\left(y_{i} \frac{\mathrm{d} w_{i}}{\mathrm{~d} z}+g_{i}^{2} \frac{\mathrm{d} \phi_{i}}{\mathrm{~d} z}\right), \quad g_{i}^{2}=\frac{1}{A_{i}} \int_{A_{i}} y^{2} \mathrm{~d} A, \quad(i=1,2), \\
V_{i}=\kappa_{i} A_{i} G_{i}\left(\frac{\mathrm{d} v}{\mathrm{~d} z}+\phi_{i}\right), \quad(i=1,2),
\end{gathered}
$$

where $\kappa_{i}$ is the shear factor of the cross-section $A_{i}(i=1,2)$ [29]. The analysis of the composite beam with interlayer slip is restricted to the case of absent axial force $N$, that is, we have

$$
N=N_{1}+N_{2}=A_{1} E_{1}\left(\frac{\mathrm{d} w_{1}}{\mathrm{~d} z}+y_{1} \frac{\mathrm{d} \phi_{1}}{\mathrm{~d} z}\right)+A_{2} E_{2}\left(\frac{\mathrm{d} w_{2}}{\mathrm{~d} z}+y_{2} \frac{\mathrm{d} \phi_{2}}{\mathrm{~d} z}\right)=0 .
$$

From equations 2.5, 2.14 it follows that

$$
\begin{gathered}
\frac{\mathrm{d} w_{1}}{\mathrm{~d} z}=\frac{A_{2} E_{2}}{\langle A E\rangle} \frac{\mathrm{d} s}{\mathrm{~d} z}-y_{1} \frac{A_{1} E_{1}}{\langle A E\rangle} \frac{\mathrm{d} \phi_{1}}{\mathrm{~d} z}-y_{2} \frac{A_{2} E_{2}}{\langle A E\rangle} \frac{\mathrm{d} \phi_{2}}{\mathrm{~d} z}, \\
\frac{\mathrm{d} w_{2}}{\mathrm{~d} z}=-\frac{A_{1} E_{1}}{\langle A E\rangle} \frac{\mathrm{d} s}{\mathrm{~d} z}-y_{1} \frac{A_{1} E_{1}}{\langle A E\rangle} \frac{\mathrm{d} \phi_{1}}{\mathrm{~d} z}-y_{2} \frac{A_{2} E_{2}}{\langle A E\rangle} \frac{\mathrm{d} \phi_{2}}{\mathrm{~d} z} .
\end{gathered}
$$



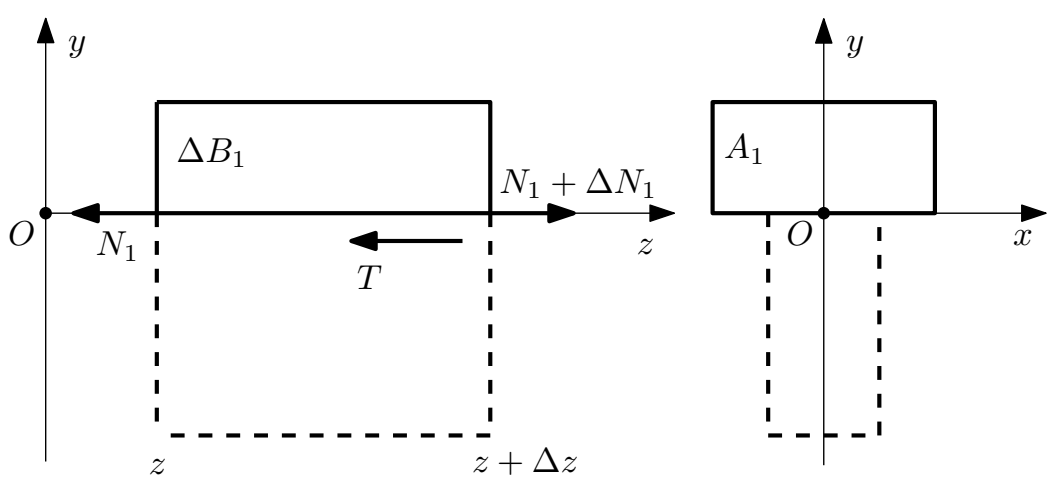

Figure 3. Equilibrium condition in the direction $z$ for a small beam element Here

$$
\langle A E\rangle=A_{1} E_{1}+A_{2} E_{2} .
$$

It is obvious that

$$
\begin{gathered}
N_{1}=\langle A E\rangle_{-1}\left[\frac{\mathrm{d} s}{\mathrm{~d} z}+y_{1} \frac{\mathrm{d} \phi_{1}}{\mathrm{~d} z}-y_{2} \frac{\mathrm{d} \phi_{2}}{\mathrm{~d} z}\right], \\
N_{2}=\langle A E\rangle_{-1}\left[-\frac{\mathrm{d} s}{\mathrm{~d} z}-y_{1} \frac{\mathrm{d} \phi_{1}}{\mathrm{~d} z}+y_{2} \frac{\mathrm{d} \phi_{2}}{\mathrm{~d} z}\right], \\
M_{1}=\langle A E\rangle_{-1}\left[y_{1} \frac{\mathrm{d} s}{\mathrm{~d} z}+c_{1} \frac{\mathrm{d} \phi_{1}}{\mathrm{~d} z}-y_{1} y_{2} \frac{\mathrm{d} \phi_{2}}{\mathrm{~d} z}\right], \\
M_{2}=\langle A E\rangle_{-1}\left[-y_{2} \frac{\mathrm{d} s}{\mathrm{~d} z}-y_{1} y_{2} \frac{\mathrm{d} \phi_{1}}{\mathrm{~d} z}+c_{2} \frac{\mathrm{d} \phi_{2}}{\mathrm{~d} z}\right],
\end{gathered}
$$

where

$$
\begin{aligned}
& \frac{1}{\langle A E\rangle_{-1}}=\frac{1}{A_{1} E_{1}}+\frac{1}{A_{2} E_{2}}, \\
& c_{1}=\frac{A_{1} E_{1}}{\langle A E\rangle_{-1}} g_{1}^{2}-y_{1}^{2} \frac{A_{1} E_{1}}{A_{2} E_{2}}, \\
& c_{2}=\frac{A_{2} E_{2}}{\langle A E\rangle_{-1}} g_{2}^{2}-y_{2}^{2} \frac{A_{2} E_{2}}{A_{1} E_{1}} .
\end{aligned}
$$

Application of the equilibrium condition for the axial forces in the beam component $B_{1}$ yields (see Figure 3 )

$$
\frac{\mathrm{d} N_{1}}{\mathrm{~d} z}-T=0
$$

In equation 2.25 $T$ is the interlayer shear force. It is assumed that $T$ is a linear function of the interlayer slip, that is

$$
T=k s,
$$




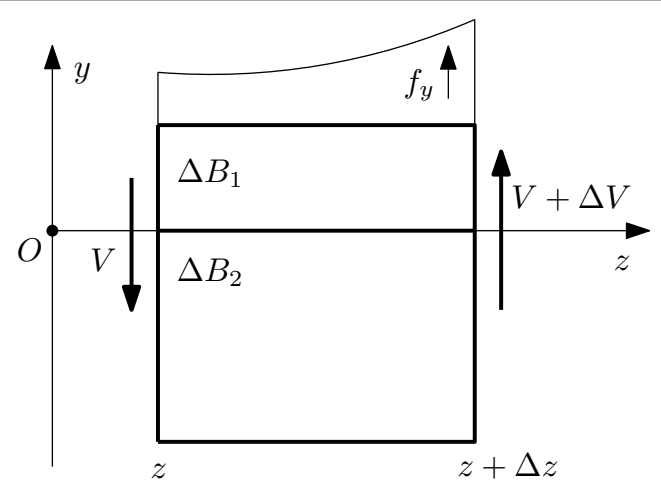

Figure 4. Shear force and vertical load on a small beam element $\Delta B=\Delta B_{1} \cup \Delta B_{2}$

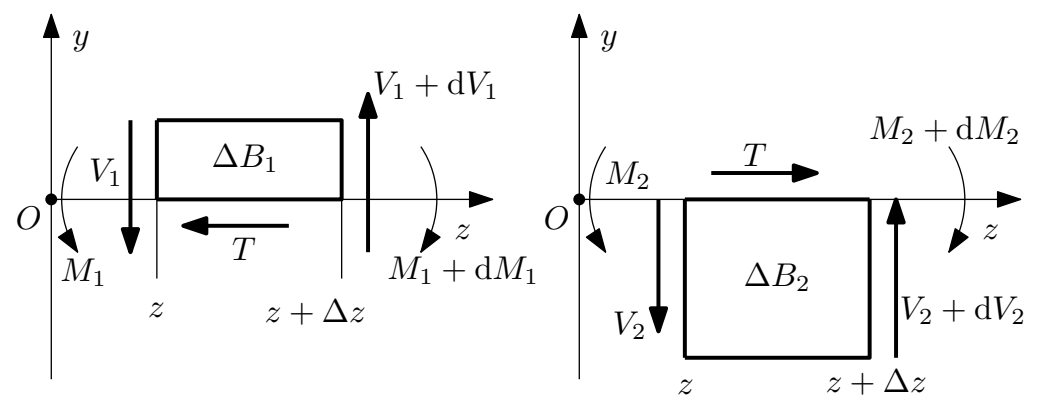

Figure 5. Shear forces and bending moments on beam elements $\Delta B_{1}$ and $\Delta B_{2}$

where $k$ is the slip modulus. From Figures 4 and Figure 5 we have the following equilibrium equations:

$$
\begin{aligned}
\frac{\mathrm{d} V}{\mathrm{~d} z}+f_{y}=0, \quad V & =V_{1}+V_{2}, \\
\frac{\mathrm{d} M_{1}}{\mathrm{~d} z}-V_{1} & =0, \\
\frac{\mathrm{d} M_{2}}{\mathrm{~d} z}-V_{2} & =0 .
\end{aligned}
$$

In order to formulate the possible boundary conditions we shall consider the virtual work of the section forces and section moments on a kinematically admissible displacement field

$$
\tilde{\mathbf{u}}_{i}=\tilde{v}(z) \mathbf{e}_{y}+\left(\tilde{w}_{i}(z)+y \tilde{\phi}_{i}(z)\right) \mathbf{e}_{z}, \quad(x, y, z) \in B_{i}, \quad(i=1,2) .
$$

A detailed computation results in

$$
W=\int_{A_{1}} \sigma_{z} \tilde{w}_{1}(z) \mathrm{d} A+\int_{A_{2}} \sigma_{z} \tilde{w}_{2}(z) \mathrm{d} A+\int_{A_{1}} y \sigma_{z} \tilde{\phi}_{1}(z) \mathrm{d} A+\int_{A_{2}} y \sigma_{z} \tilde{\phi}_{2}(z) \mathrm{d} A+
$$


Table 1. Classical boundary conditions

\begin{tabular}{|c|c|}
\hline Type & Boundary condition \\
\hline & $v=0, s=0, \phi_{1}=0, \phi_{2}=0$ (kinematical boundary \\
conditions)
\end{tabular}

$+\int_{A} \tau_{y z} \tilde{v}(z) \mathrm{d} A=N_{1} \tilde{w}_{1}+N_{2} \tilde{w}_{2}+M_{1} \tilde{\phi}_{1}+M_{2} \tilde{\phi}_{2}+V \tilde{v}=N_{1} \tilde{s}+M_{1} \tilde{\phi}_{1}+M_{2} \tilde{\phi}_{2}+V \tilde{v}$

where

$$
\tilde{s}=\tilde{w}_{1}(z)-\tilde{w}_{2}(z) .
$$

From equation (2.31) we obtain the possible combinations of the boundary conditions at the end cross-section

$V$ or $v$ may be prescribed,

$N_{1}$ or $s$ may be prescribed,

$M_{1}$ or $\phi_{1}$ may be prescribed,

$M_{2}$ or $\phi_{2}$ may be prescribed. 
We remark that some classical boundary conditions are listed on the basis of equations 2.332 .36 in Table 1. It can be checked by utilizing equations 2.18 and 2.20 that the boundary conditions for a simply supported end and a free end are

$$
\frac{\mathrm{d} s}{\mathrm{~d} z}=0, \quad \frac{\mathrm{d} \phi_{1}}{\mathrm{~d} z}=0, \quad \frac{\mathrm{d} \phi_{2}}{\mathrm{~d} z}=0
$$

The combination of equations 2.13, 2.18, 2.20, 2.21), 2.26 with equations 2.25, 2.27, 2.28, 2.29 yields a system of linear differential equations for the functions $v=v(z), s=s(z), \phi_{1}=\phi_{1}(z)$ and $\phi_{2}=\phi_{2}(z)$

$$
\begin{gathered}
\frac{\mathrm{d}^{2} s}{\mathrm{~d} z^{2}}+y_{1} \frac{\mathrm{d}^{2} \phi_{1}}{\mathrm{~d} z^{2}}-y_{2} \frac{\mathrm{d}^{2} \phi_{2}}{\mathrm{~d} z^{2}}-\frac{k}{\langle A E\rangle_{-1}} s=0, \\
y_{1} \frac{\mathrm{d}^{2} s}{\mathrm{~d} z^{2}}+c_{1} \frac{\mathrm{d}^{2} \phi_{1}}{\mathrm{~d} z^{2}}-y_{1} y_{2} \frac{\mathrm{d}^{2} \phi_{2}}{\mathrm{~d} z^{2}}-\kappa_{1} \frac{G_{1} A_{1}}{\langle A E\rangle_{-1}}\left(\frac{\mathrm{d} v}{\mathrm{~d} z}+\phi_{1}\right)=0, \\
-y_{2} \frac{\mathrm{d}^{2} s}{\mathrm{~d} z^{2}}-y_{1} y_{2} \frac{\mathrm{d}^{2} \phi_{1}}{\mathrm{~d} z^{2}}+c_{2} \frac{\mathrm{d}^{2} \phi_{2}}{\mathrm{~d} z^{2}}-\kappa_{2} \frac{G_{2} A_{2}}{\langle A E\rangle_{-1}}\left(\frac{\mathrm{d} v}{\mathrm{~d} z}+\phi_{2}\right)=0, \\
\kappa_{1} G_{1} A_{1}\left(\frac{\mathrm{d}^{2} v}{\mathrm{~d} z^{2}}+\frac{\mathrm{d} \phi_{1}}{\mathrm{~d} z}\right)+\kappa_{2} G_{2} A_{2}\left(\frac{\mathrm{d}^{2} v}{\mathrm{~d} z^{2}}+\frac{\mathrm{d} \phi_{2}}{\mathrm{~d} z}\right)=-f_{y}(z) .
\end{gathered}
$$

\section{Solution FOR A SIMPLy SUPPORTED BEAM}

For a simply supported beam we look for the solution of the ordinary differential equation system 2.38 2.41) in the following form:

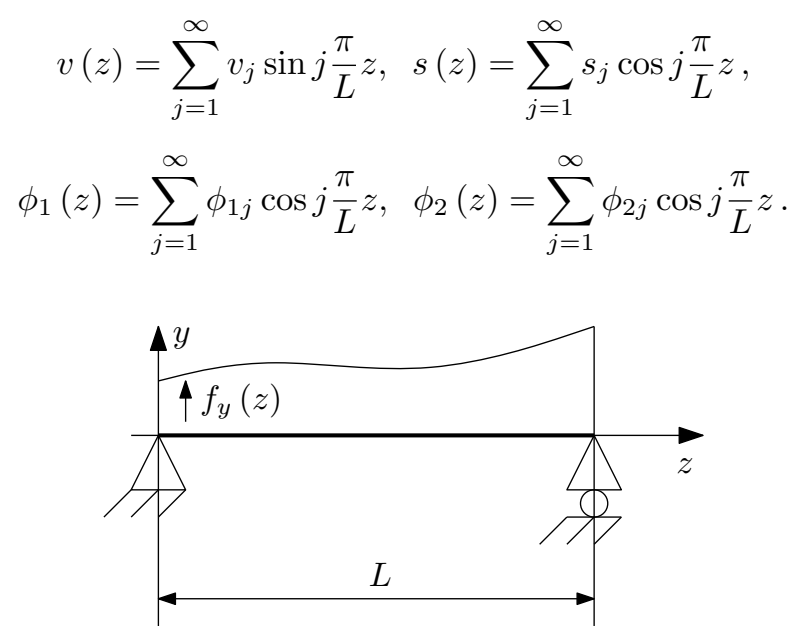

Figure 6. Simply supported beam

These functions satisfy the boundary conditions

$$
v=0, \quad N_{1}=0, \quad M_{1}=0, \quad M_{2}=0
$$


for arbitrary values of $v_{j}, s_{j}, \phi_{1 j}, \phi_{2 j},(j=1,2, \ldots)$ at both ends of the beam. Substitution of $v=v(z), s=s(z), \phi_{1}=\phi_{1}(z)$ and $\phi_{2}=\phi_{2}(z)$ into the ODE system 2.38 2.41 results in a system of linear equations for $v_{j}, s_{j}, \phi_{1 j}, \phi_{2 j},(j=1,2, \ldots)$. This can be written as

$$
\mathbf{C}_{j} \mathbf{x}_{j}=\mathbf{f}_{j}, \quad(j=1,2, \ldots)
$$

where

$$
\begin{gathered}
\mathbf{C}_{j}=\left[c_{j p q}\right], \quad(p, q=1,2,3,4) \\
c_{j 11}=-\left(j \frac{\pi}{L}\right)^{2}-\frac{k}{\langle A E\rangle_{-1}}, \quad c_{j 12}=-y_{1}\left(j \frac{\pi}{L}\right)^{2}, c_{j 13}=y_{2}\left(j \frac{\pi}{L}\right)^{2}, \quad c_{j 14}=0 \\
c_{j 21}=-y_{1}\left(j \frac{\pi}{L}\right)^{2}, \quad c_{j 22}=-c_{1}\left(j \frac{\pi}{L}\right)^{2}+\frac{\kappa_{1} G_{1} A_{1}}{\langle A E\rangle_{-1}}, \\
c_{j 23}=y_{1} y_{2}\left(j \frac{\pi}{L}\right)^{2}, c_{j 24}=-\frac{\kappa_{1} G_{1} A_{1}}{\langle A E\rangle_{-1}} j \frac{\pi}{L} \\
c_{j 31}=y_{2}\left(j \frac{\pi}{L}\right)^{2}, \quad c_{j 32}=y_{1} y_{2}\left(j \frac{\pi}{L}\right)^{2}, \\
c_{j 33}=-c_{2}\left(j \frac{\pi}{L}\right)^{2}+\frac{\kappa_{2} G_{2} A_{2}}{\langle A E\rangle_{-1}}, c_{j 34}=-\frac{\kappa_{2} G_{2} A_{2}}{\langle A E\rangle_{-1}} j \\
c_{j 41}=0, c_{j 42}=-\kappa_{1} G_{1} A_{1} j \frac{\pi}{L}, c_{j 43}=-\kappa_{2} G_{2} A_{2} j \frac{\pi}{L} \\
c_{j 44}=-\left(\kappa_{1} G_{1} A_{1}+\kappa_{2} G_{2} A_{2}\right)\left(j \frac{\pi}{L}\right)^{2} \\
\mathbf{x}_{j}^{T}=\left[s_{j}, \phi_{1 j}, \phi_{2 j}, v_{j}\right] \\
\mathbf{f}_{j}^{T}=\left[0,0,0, f_{j}\right] .
\end{gathered}
$$

In formula $3.10 f_{j}$ is defined as

$$
f_{j}=\frac{2}{L} \int_{0}^{L} f_{y}(z) \sin j \frac{\pi}{L} z \mathrm{~d} z, \quad(j=1,2, \ldots) .
$$

Assume that the beam is subjected to the load

$$
f_{y}(z)=-f\left[H\left(z-\frac{L}{2}+\frac{l}{2}\right)-H\left(z-\frac{L}{2}-\frac{l}{2}\right)\right]
$$

where $H=H(z)$ is the Heaviside function. The type of applied load given by equation (3.12) and the meanings of $f$ and $l(0 \leq l \leq L)$ are shown in Figure 7 For this load the Fourier coefficients $f_{j}$ are given by the following equation:

$$
f_{j}=-\frac{4 f}{j \pi} \sin \left(\frac{j \pi}{2}\right) \sin \left(\frac{j \pi}{2} \frac{l}{L}\right),(j=1,2, \ldots) .
$$




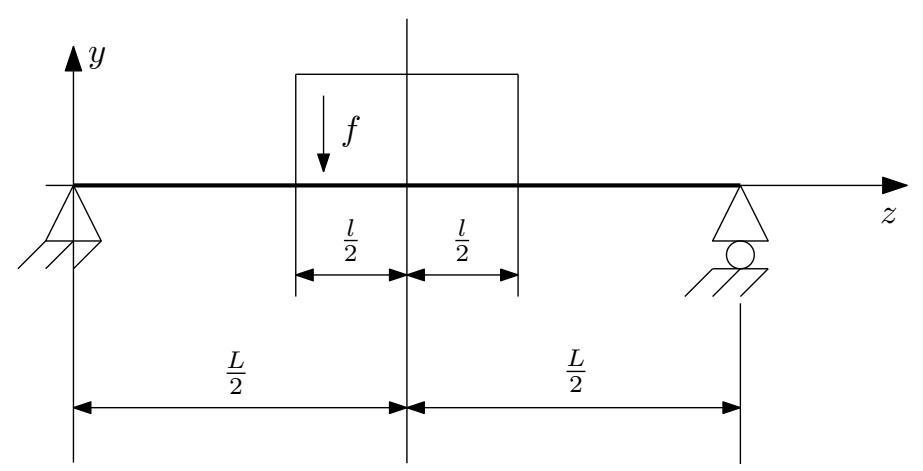

Figure 7. Illustration of applied load

\section{Free VIBRATIONS OF A SIMPLY SUPPORTED BEAM}

To formulate the governing equations of the free vibrations we introduce the inertia forces into equations (2.27), (2.28) and (2.29). It is obvious that all physical quantities depend on time $t$ too, that is $v=v(z, t), s=s(z, t), \phi_{1}=\phi_{1}(z, t), \phi_{2}=\phi_{2}(z, t)$, etc. Assuming free vibrations we obtain

$$
v=\bar{v}(z) \sin \omega t, \quad s=\bar{s}(z) \sin \omega t, \quad \phi_{1}=\bar{\phi}_{1}(z) \sin \omega t, \quad \phi_{2}=\bar{\phi}_{2}(z) \sin \omega t,
$$

where $\omega$ is a natural frequency and $\bar{v}(z), \ldots, \bar{\phi}_{2}(z)$ are the unknown amplitude functions. The inertia force from the vertical motion is of the form

$$
f_{y}(z, t)=-\left(\rho_{1} A_{1}+\rho_{2} A_{2}\right) \frac{\partial^{2} v}{\partial t^{2}}=\omega^{2}\left(\rho_{1} A_{1}+\rho_{2} A_{2}\right) \bar{v}(z) \sin \omega t .
$$

The inertia couples from the cross-sectional rotations are

$$
\begin{aligned}
& m_{1}(z, t)=-\rho_{1} I_{1} \frac{\partial^{2} \phi_{1}}{\partial t^{2}}=\omega^{2} \rho_{1} I_{1} \bar{\phi}_{1}(z) \sin \omega t, \\
& m_{2}(z, t)=-\rho_{2} I_{2} \frac{\partial^{2} \phi_{2}}{\partial t^{2}}=\omega^{2} \rho_{2} I_{2} \bar{\phi}_{2}(z) \sin \omega t .
\end{aligned}
$$

In equations 4.2 4.4 $\rho_{i}(i=1,2)$ is the mass density and

$$
I_{i}=\int_{A_{i}} y^{2} \mathrm{~d} A, \quad(i=1,2) .
$$

Upon substitution of equation 4.2 into equation 2.27) we obtain

$$
\kappa_{1} G_{1} A_{1}\left(\frac{\mathrm{d} \bar{v}}{\mathrm{~d} z}+\bar{\phi}_{1}\right)+\kappa_{2} G_{2} A_{2}\left(\frac{\mathrm{d} \bar{v}}{\mathrm{~d} z}+\bar{\phi}_{2}\right)+\omega^{2}\left(\rho_{1} A_{1}+\rho_{2} A_{2}\right) \bar{v}=0 .
$$

Combination of equations 2.28 and $(2.29$ with equations 4.3 and 4.4 yields the following two equations:

$$
y_{1} \frac{\mathrm{d}^{2} \bar{s}}{\mathrm{~d} z^{2}}+c_{1} \frac{\mathrm{d}^{2} \bar{\phi}_{1}}{\mathrm{~d} z^{2}}-y_{1} y_{2} \frac{\mathrm{d}^{2} \bar{\phi}_{2}}{\mathrm{~d} z^{2}}-\frac{\kappa_{1} G_{1} A_{1}}{\langle A E\rangle_{-1}}\left(\frac{\mathrm{d} \bar{v}}{\mathrm{~d} z}+\bar{\phi}_{1}\right)+\omega^{2} \frac{\rho_{1} I_{1}}{\langle A E\rangle_{-1}} \bar{\phi}_{1}(z)=0,
$$




$$
-y_{2} \frac{\mathrm{d}^{2} \bar{s}}{\mathrm{~d} z^{2}}-y_{1} y_{2} \frac{\mathrm{d}^{2} \bar{\phi}_{1}}{\mathrm{~d} z^{2}}+c_{2} \frac{\mathrm{d}^{2} \bar{\phi}_{2}}{\mathrm{~d} z^{2}}-\frac{\kappa_{2} G_{2} A_{2}}{\langle A E\rangle_{-1}}\left(\frac{\mathrm{d} \bar{v}}{\mathrm{~d} z}+\bar{\phi}_{2}\right)+\omega^{2} \frac{\rho_{2} I_{2}}{\langle A E\rangle_{-1}} \bar{\phi}_{2}(z)=0 .
$$

We introduce the mass matrix $\mathbf{M}$ by the following definition

$$
\begin{gathered}
\mathbf{M}_{j}=\left[m_{j p q}\right], \quad(p, q=1,2,3,4), \quad i=1,2, \ldots, \\
m_{j 11}=m_{j 12}=m_{j 13}=m_{j 14}=0, \\
m_{j 21}=m_{j 23}=m_{j 24}=0, \quad m_{j 22}=-\frac{\rho_{1} I_{1}}{\langle A E\rangle_{-1}}, \\
m_{j 31}=m_{j 32}=m_{j 34}=0, \quad m_{j 33}=-\frac{\rho_{2} I_{2}}{\langle A E\rangle_{-1}}, \\
m_{j 41}=m_{j 42}=m_{j 43}=0, \quad m_{j 44}=\left(\rho_{1} A_{1}+\rho_{2} A_{2}\right) .
\end{gathered}
$$

Further let

$$
\mathbf{X}_{j}^{T}=\left[\bar{s}_{j}, \bar{\phi}_{1 j}, \bar{\phi}_{2 j}, \bar{v}_{j}\right] .
$$

For the free vibrations of simply supported composite beams with weak shear connection we assume that

$$
\begin{gathered}
\bar{v}(z)=\sum_{j=1}^{\infty} \tilde{v}_{j} \sin j \frac{\pi}{L} z, \bar{s}(z)=\sum_{j=1}^{\infty} \tilde{s}_{j} \cos j \frac{\pi}{L} z \\
\bar{\phi}_{1}(z)=\sum_{j=1}^{\infty} \tilde{\phi}_{1 j} \cos j \frac{\pi}{L} z, \bar{\phi}_{2}(z)=\sum_{j=1}^{\infty} \tilde{\phi}_{2 j} \cos j \frac{\pi}{L} z .
\end{gathered}
$$

A comparison with equations (3.1) shows that the boundary conditions $(3.2)$ are again satisfied. By repeating the line of thought resulting in equation $(3.3)$ the eigenvalue problem for the free vibrations of simply supported two-layer beams with flexible shear connection can be formulated as

$$
\left(\mathbf{C}_{j}+\omega^{2} \mathbf{M}_{j}\right) \mathbf{X}_{j}=\mathbf{0}, \quad(j=1,2 \ldots) .
$$

For each $j$ we have three different natural frequencies. The smaller value of $\omega_{j}^{2}$ corresponds to the bending deformation mode and the other two values of $\omega_{j}^{2}$ correspond to the shear deformation modes. For the Euler-Bernoulli and Euler-Bernoulli-Rayleigh beams we have only bending deformation mode and the natural frequencies can be obtained - see 26 - from the following equation

$$
\Omega_{j}^{2}=\frac{\langle I E\rangle\left[\left(\frac{j \pi}{L}\right)^{4}+\left(\frac{j \Gamma}{L}\right)^{2}\right]\left(\frac{j \pi}{L}\right)^{2}}{\left[m+I_{m}\left(\frac{j \pi}{L}\right)^{2}\right]\left[\frac{k}{\langle A E\rangle_{-1}}+\left(\frac{j \pi}{L}\right)^{2}\right]},
$$

where

$$
\begin{gathered}
\langle I E\rangle=E_{i} \int_{A_{i}}\left(y-y_{i}\right)^{2} \mathrm{~d} A, \quad(i=1,2), \quad m=\rho_{1} A_{1}+\rho_{2} A_{2}, \\
\Gamma^{2}=\frac{k\{I E\}}{\langle A E\rangle_{-1}\langle I E\rangle}, \quad\{I E\}=\langle I E\rangle+\left(y_{1}-y_{2}\right)^{2}\langle A E\rangle_{-1}, \\
I_{m}=0 \quad \text { (Euler-Bernoulli beam) },
\end{gathered}
$$




$$
I_{m}=\sum_{i=1}^{2}\left[\rho_{i} \int_{A_{i}}\left(y-y_{i}\right)^{2} \mathrm{~d} A+y_{i}^{2} \rho_{i} A_{i}\right] \quad \text { (Euler-Bernoulli-Rayliegh beam) . }
$$

\section{Numerical EXAMPLES}

5.1. Simply supported beam loaded by uniform distributed force. This example is taken from paper [18] by Schnabl et al. The simply supported beam, its cross-section and the applied load are shown in Figure 8. The following data are used $h_{1}=0.2[\mathrm{~m}], h_{2}=0.3[\mathrm{~m}], b=0.3[\mathrm{~m}], E_{1}=1.2 \times 10^{10}[\mathrm{~Pa}], E_{2}=1.2 \times 10^{10}[\mathrm{~Pa}]$, $G_{1}=8 \times 10^{8}[\mathrm{~Pa}], G_{2}=1.2 \times 10^{9}[\mathrm{~Pa}], f=5 \times 10^{4}[\mathrm{~N} / \mathrm{m}], k=2.43 \times 10^{6}[\mathrm{~Pa}]$, $L=2.5[\mathrm{~m}], \kappa_{1}=\kappa_{2}=5 / 6$. The functions $v=v(z)$ and $s=s(z)$ are shown in Figures 9 and 10 . The functions $\phi_{1}=\phi_{1}(z)$ and $\phi_{2}=\phi_{2}(z)$ are also presented in graphical format - see Figure 11. A comparison of the deflection $v\left(\frac{L}{2}\right)$ and the slip $s(0)$ with the results obtained by Schnabl et al. 18 is given in Table 2
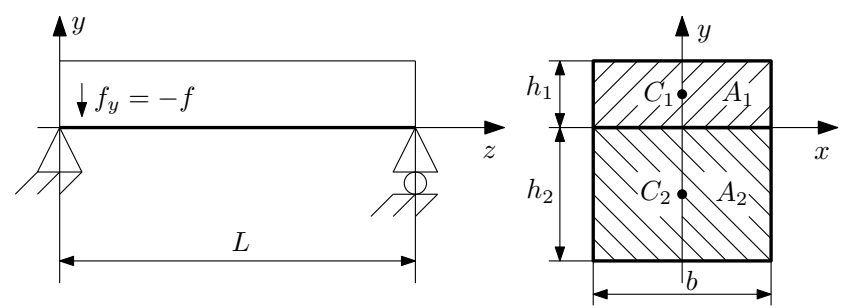

Figure 8. Simply supported composite beam with uniform load

Table 2. Comparison of deflection and slip

\begin{tabular}{|c|c|c|}
\hline & \begin{tabular}{l|l|l} 
paper & (FEM) \\
\end{tabular} & present paper \\
\hline$\overline{v(L / 2)[\mathrm{m}]}$ & -0.00271026 & -0.0027082964 \\
\hline$s(0)[\mathrm{m}]$ & -0.00077293 & -0.0007713293 \\
\hline
\end{tabular}

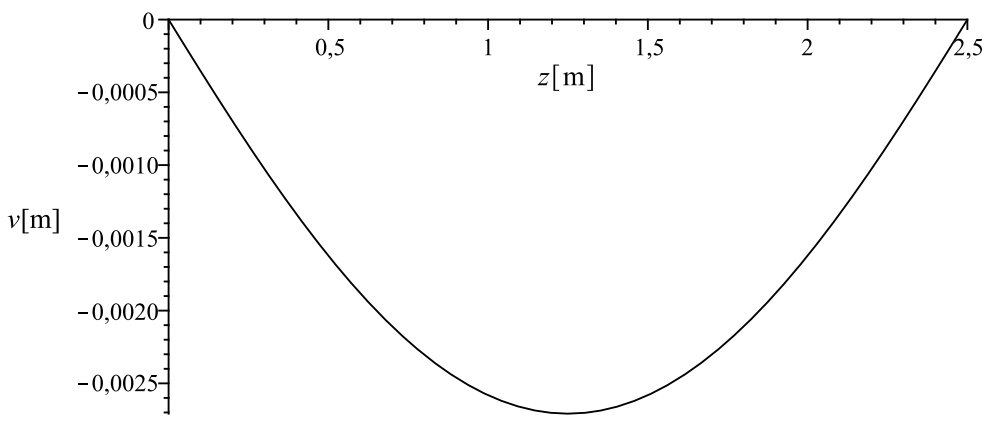

Figure 9. The plot of $v(z)$ 


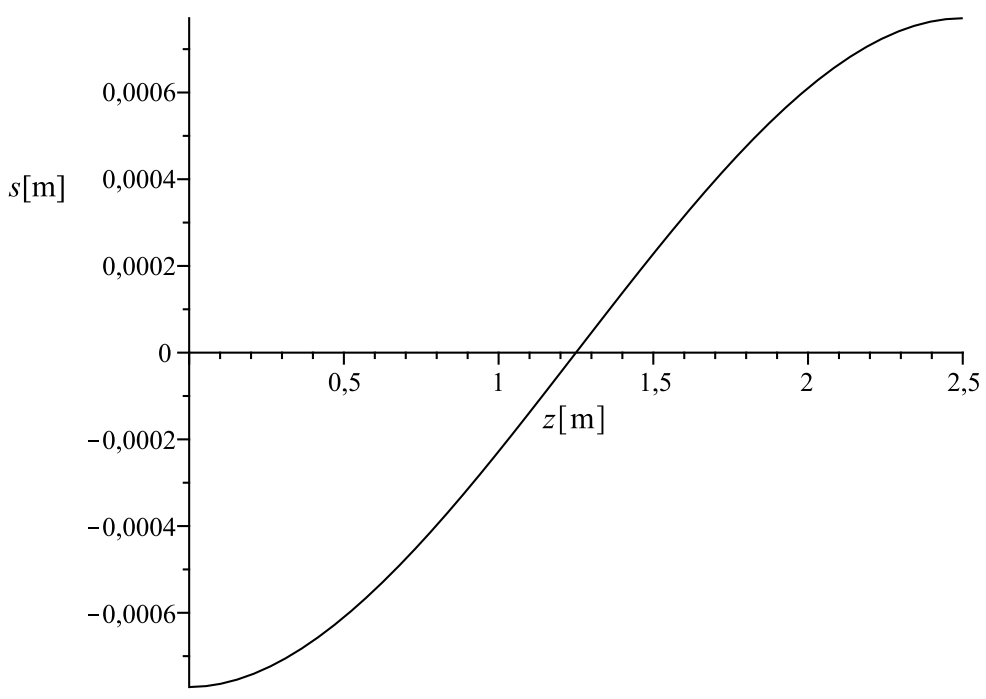

Figure 10. The plot of $s(z)$

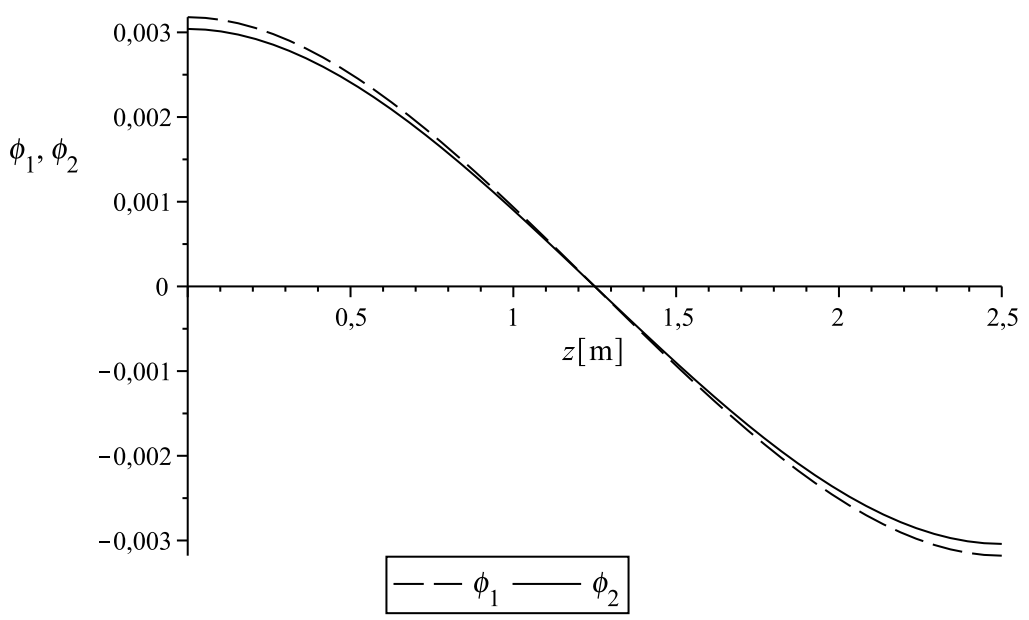

Figure 11. The plots of $\phi_{1}(z)$ and $\phi_{2}(z)$ 


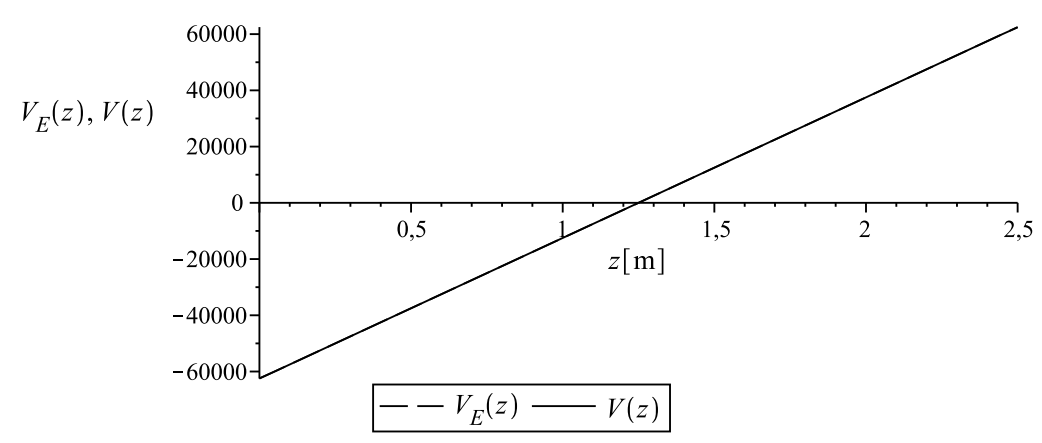

Figure 12. The shear force functions

For completeness Figure 12 shows the shear force

$$
V_{E}(z)=f\left(z-\frac{L}{2}\right) \text {. }
$$

The shear force function $V=V(z)$ is computed from the deflection and the crosssectional rotation by utilizing equations $2.13,2.27)_{1}$

$$
V(z)=\kappa_{1} G_{1} A_{1}\left(\frac{\mathrm{d} v}{\mathrm{~d} z}+\phi_{1}\right)+\kappa_{2} G_{2} A_{2}\left(\frac{\mathrm{d} v}{\mathrm{~d} z}+\phi_{2}\right) .
$$

This function is also shown in Figure 12 The curves $V_{E}(z)$ and $V(z)$ coincide and this fact is evidence for the accuracy of the presented solutions.

5.2. Natural frequencies of the free vibrations. The data for this example are the same as those in Example 5.1. The densities should also be given: $\rho_{1}=$ $5000\left[\mathrm{~kg} / \mathrm{m}^{3}\right], \rho_{2}=7000\left[\mathrm{~kg} / \mathrm{m}^{3}\right]$. The results we have obtained for the natural frequencies are listed in Table 3

Table 3. Natural frequencies

\begin{tabular}{|c|c|c|c|c|c|}
\hline $\mathrm{j}$ & Euler-Bernoulli & Euler-Bernoulli- & \multicolumn{3}{|c|}{ Timoshenko } \\
\cline { 4 - 6 } & & Rayleigh & Bending & Shear & Shear \\
\hline 1 & 155.290664 & 152.6194487 & 151.4225922 & 2338.54532 & 8394.253453 \\
\hline 2 & 622.4043575 & 582.6170364 & 504.1041551 & 2901.662787 & 3724.202344 \\
\hline 3 & 1400.929022 & 1220.369015 & 941.8521016 & 3635.127974 & 4202.393801 \\
\hline 4 & 2490.863793 & 1991.111501 & 1414.604281 & 4451.987908 & 4779.655958 \\
\hline 5 & 3892.20856 & 2836.587389 & 1902.205348 & 5291.520318 & 5444.286196 \\
\hline
\end{tabular}

\section{Conclusions}

In this paper an analytical model has been developed to analyse the deformation of composite beams with weak shear connections. Timoshenko beam theory is used, assuming that the layers have different cross-sectional rotations. Analytical solutions 
for deflection, slip and cross-sectional rotations are derived. The eigenfrequencies of free vibrations of a simply supported beam are also computed. The presented solutions are based on the representations of applied load, deflection, slip and crosssectional rotations by Fourier series. The numerical results we have obtained are compared with a FEM solution and good agreement has been found.

Acknowledgements. This research was carried out as a part of TÁMOP-4.2.1.B10/2KONV-2010-0001 project with support by European Union and co-financed by European Social Found.

\section{REFERENCES}

1. Granholm, H.: On composite beams and columns with special regard to nailed structures. Trans. No. 88. Chalmers University of Technology, Göteborg, Sweden, 1949. (in Swedish).

2. Pleskov, F. P.: Theoretical Studies of Composite Wood Structures. Soviet Union, 1952. (in Russian).

3. Stüssi, F.: Zusammengesetzte Vollwandträger. IABSE Publications, 8, (1947), 249-269.

4. Newmark, N. M., Siess, C. P., and Viest, I. M.: Test and analysis of composite beam with incomplete interaction. Proc. Soc. Exp. Stress Anal., 9, (1951), 75-92.

5. Goodman, I.: Layered wood system with interlayer slip. Ph. D. thesis, University of California, Berkeley, CA, 1967.

6. Goodman, I. and Popov, E.: Layered beam system with inter-layer slip. Journal of the Structural Engineering, Division ASCE, 94(11), (1968), 2537-2547.

7. Goodman, I. and Popov, E.: Layered wood system with inter-layer slip. Wood Science, 1(3), (1969), 148-158.

8. Thomson, F., Goodman, I., and Vanderbilt, M.: Finite element analysis of layered wood systems. Journ. of Struct., Division ASCE, 101(12), (1975), 2659-2672.

9. Girhammar, U. A. and Gopu, V.: Composite beam-columns with interlayer slip exact analysis. Journ. Struct. Eng., 119(4), (1993), 1265-1282.

10. Girhammar, U. A. and Pan, D. H.: Exact static analysis of partially composite beams and beam-columns. Int. Journ. Mech. Sci., 49(2), (2007), 239-255.

11. Girhammar, U. A.: A simplified analysis method for composite beams with interlayer slip. Int. Journ. Mech. Sci., 51(7), (2009), 515-530.

12. ECSEDI, I. and BAKsA, A.: Static analysis of composite beams with weak shear connection. Appl. Math. Mod., (35), (2011), 1739-1750.

13. Ranzi, G., Bradford, M., and Uy, B.: A direct stiffness analysis of composite beam with partial interaction. Int. Journ. Num. Methods Eng., 61(5), (2004), 657-672.

14. WANG, C.: Deflection of steel-concrete composite beams with partial shear interaction. Journ. Struct. Eng., 124(10), (1998), 1159-1165.

15. Xu, R. and Wu, Y.: Static, dynamic and buckling analysis of partial interaction composite members using Timoshenko beam theory. Int. Journ. Mech. Sci., 49(10), (2007), $1139-1155$.

16. Nguyen, Q., Martinelli, E., and Huiaj, M.: Derivation of the exact stiffness matrix for a two-layer Timoshenko beam element with partial interaction. Eng. Struct., 33(2), (2011), 298-307. 
17. Nguyen, Q., Huiaj, M., and Guezouli, S.: Exact finite element model for sheardeformable two-layer beams with discrete shear connection. Finite Element in Analysis and Design, 47(7), (2011), 718-727.

18. Schnabl, S., Saje, M., Turk, G., and Planinc, I.: Locking free two-layer Timoshenko beam element with interlayer slip. Finite Elements in Analysis and Design, 43(9), (2007), 705-714.

19. Lin, X. and Zhang, Y.: A novel one-dimensional two-node shear-flexible layered composite beam element. Finite Elements in Analysis and Design, 47(7), (2011), 676-682.

20. Jiang, S., Zeng, X., and Zhou, D.: Novel two-node linear composite beam element with both interface slip and shear deformation into consideration: Formulation and validation. Int. Journ. Mech. Sci, 85, (2014), 110-119.

21. Schnabl, S., Saje, M., Turk, G., and Planinc, I.: Analytical solution of two-layer beam taking into account interlayer slip and shear deformation. Journ. Struct. Eng. ASCE, 133(6), (2007), 886-894.

22. Saje, M., Cas, B., and Planinc, I.: Non-linear finite element analysis of composite planar frames with an interlayer slip. Comp. Struc., 82(23-26), (2004), 1901-1912.

23. Girhammar, U. A. and Pan, D.: Dynamic analysis of composite members with interlayer slip. Int. Journ. Solids and Struct., 30(6), (1993), 797-823.

24. Wu, Y. F., Xu, R., and Chen, W.: Free vibrations of the partial-interaction composite members with axial force. Journ. Sound and Vib., 299(4-5), (2007), 1074-1093.

25. NGuyen, Q. H., Huiaj, M., and Gronec, P. L.: Analytical approach for free vibration analysis of two-layer Timoshenko beams with interlayer slip. Journ. Sound and Vib., 331(3), (2012), 2949-2961.

26. Ecsedi, I. and Dluhi, K.: Linear analysis of composite beam-columns with weak shear connection. In MicroCAD 2005 International Scientific Conference, 2005, pp. 37-42. Section G: Applied Mechanics, Modern Numerical Methods.

27. Sokolnikoff, I. S.: Mathematical Theory of Elasticity. McGraw-Hill, New York, 2nd edn., 1956.

28. Timoshenko, S. P. and Goodier, J. N.: Theory of Elasticity. McGraw-Hill, New York, 3rd edn., 1970.

29. Cowper, G. R.: The shear coefficient in Timoshenko's beam theory. Journ. Appl. Mech., 33(2), (1966), 335-340. 